Cahiers Société

\title{
La saisie du social-historique : création et méthode chez Castoriadis
}

\section{Geneviève Gendreau}

Numéro 1, mai 2019

Castoriadis et les sciences sociales

URI : https://id.erudit.org/iderudit/1068423ar

DOI : https://doi.org/10.7202/1068423ar

Aller au sommaire du numéro

\section{Éditeur(s)}

Collectif Société

ISSN

2562-5373 (imprimé)

2562-5381 (numérique)

Découvrir la revue

Citer cet article

Gendreau, G. (2019). La saisie du social-historique : création et méthode chez Castoriadis. Cahiers Société, (1), 125-151. https://doi.org/10.7202/1068423ar
Résumé de l'article

Ce texte explore les propositions épistémologiques de Castoriadis, d’abord à partir de sa critique des sciences sociales, qui montre l'occultation systématique dont le social-historique a fait l'objet. Contre cet héritage des sciences sociales seront examinées ses propositions faisant de l'imaginaire social le lieu de l'institution de toute société. Si Castoriadis ne récuse en rien l'apport des méthodes explicatives et compréhensives en sciences sociales, il spécifie toutefois la région de l'être du social-historique qu'elles sont à même d'éclairer : ses éléments ensidiques, dans le cas des premières ; ses significations imaginaires sociales, dans le cas des secondes. Toutefois, son originalité pour les sciences sociales apparaît dans la nécessité d'une élucidation du noyau même des significations sociales, laquelle ne saurait être pensée en dehors de ses liens, politiques, au projet d'autonomie, ni en dehors de son ontologie de la création/destruction radicale. 


\title{
La saisie du social-historique : création et méthode chez Castoriadis
}

\author{
Geneviève GENDREAU \\ Université d'Ottawa
}

Castoriadis unifie la société et l'histoire sous l'expression de «social-historique ». Bien plus qu'une posture rhétorique, il s'agit, reprenant l'une de ses expressions, d'une « autoposition », de nature tout à la fois politique, ontologique et épistémologique, concernant le mode d'être de la société. Il souhaite par là souligner, d'abord, la consubstantialité de la société et de l'histoire et donc, négativement, l'inanité des découpages entre dimensions synchronique et diachronique. Ceci au sens bien précis où toute société n'est pas simplement « dans le temps », comme si le temps lui était extérieur, mais au sens où toute société est son déploiement dans le temps. Il insiste également sur la nécessité de concevoir le social-historique comme étant le siège d'une puissance de création radicale immotivée, niveau d'être qui est à lui-même son propre modèle. Castoriadis a consacré une bonne partie de son œuvre à développer les implications de ce niveau d'être particulier et, comme les textes de Stéphane Vibert et de Thibault Tranchant ici le démontrent bien, à critiquer ce qu'il a nommé la «pensée héritée », philosophie et sciences sociales confondues, pour leur occultation systématique de ce mode d'être.

À vrai dire, Castoriadis a amorcé son questionnement sur l'être de la société très tôt, avant même l'époque de Socialisme ou Barbarie, ce dont l'ouvrage Histoire et création, publié en 2009 et issu de manuscrits inédits rédigés entre 1945 et 1967 provenant de sa dissertation doctorale inachevée, portant sur Max Weber, témoigne ${ }^{1}$. En réponse, ensuite, à la philosophie déterministe de l'histoire marxiste et à son anthropologie philosophique productiviste et économiciste, on trouve, dès les premières pages du texte " Marxisme et théorie révolutionnaire » paru d'abord dans Socialisme ou Barbarie, puis repris en première partie de L'institution imaginaire de la société, paru en 1975, l'affirmation selon laquelle il avait choisi de rester révolutionnaire plutôt que marxiste, c'est-à-dire de manifester sa « fidélité au projet d'une transformation radicale de la société, qui exige d'abord que l'on comprenne ce que l'on veut

${ }^{1}$ Cornelius Castoriadis, Histoire et création : textes philosophiques inédits, 1945-1967, Paris, Seuil, 2009. 
transformer ${ }^{2}$ ». C'est bien cette « exigence de compréhension » du social-historique tel qu'il est en lui-même qui nous occupera ici.

Ce texte se concentrera dans un premier temps sur la conception castoriadienne du social-historique. Nous aborderons d'abord et avant tout les implications du niveau d'être du social-historique sur les rapports entre le fonctionnel et l'imaginaire, afin d'expliciter les manières de rendre compte de l'«effectif»d'une société par l'analyse, pour ensuite nous tourner spécifiquement vers la question épistémologique de ses modes de saisie, prescrits et proscrits. Peut-on, et si oui, comment, "rendre compte et raison» d'une société ? Quel sens y a-t-il à affirmer, comme le fait Castoriadis, d'une part, que le recours à la causalité en sciences sociales est " légitime ", mais que celle-ci est, par ailleurs, foncièrement inapte à saisir le socialhistorique en tant que tel ? De même, comment faut-il comprendre son affirmation selon laquelle il ne saurait y avoir de «méthode» à même de le saisir, alors qu'il énonce par ailleurs les grandes étapes du processus de compréhension des sociétés? Plus encore, alors qu'il exprime sans ambages le but que devrait selon lui viser toute tentative d'explicitation des sociétés, en appelant ainsi à une refonte majeure des sciences sociales, tant dans leur contenu théorique que méthodologique, ainsi que dans leur portée normative et proprement politique? Une fois la nature ontologique du social-historique et ses implications épistémologiques établies, nous reviendrons, dans un troisième temps, sur le paradigme de la création/destruction ontologique, puisque ce dernier informe tout le projet de compréhension totale et d'élucidation qu'il propose en réponse à la pensée héritée. Ce sera alors l'occasion de saisir la création historique castoriadienne comme déployant un double mouvement, à proprement parler indissociable, posant le nouveau comme étant à la fois immotivé et foncièrement circulaire.

\section{Le social-historique comme niveau d'être sui generis}

\section{Fonctionnalité, effectivité et imaginaire}

Pour Castoriadis, le fait que chaque société s'auto-institue en fonction de son imaginaire est au cour de l'analyse du social-historique. Le terme d' « institution » est entendu ici au sens le plus large qu'on puisse concevoir, dans la foulée de Mauss, ainsi que des usages de la notion de Stiftung3 dans la phénoménologie husserlienne, reprise

\footnotetext{
2 Cornelius Castoriadis, L'institution imaginaire de la société, Paris, Seuil, 1999, p. 21.

3 Dans son premier texte consacré à Merleau-Ponty, «Le dicible et l'indicible. Hommage à MerleauPonty », datant de 1971, Castoriadis discute la notion de Stiftung, soulignant l'actualité et l'effectivité, à tout moment de l'existence sociale, de l'origine instituante elle-même, de sorte que celle-ci ne puisse être rabattue ni sur le moment de l'origine (Urstiftung) ni sur la succession des différentes institutions (Stiftung) dans le temps. Nous voyons donc dans cette remarque poindre le thème récurrent de la création du social-historique par lui-même, se manifestant comme auto-institution et travail incessant, toujours actuel et actualisé, de l'instituant. Cf. Les carrefours du labyrinthe, I, Paris, Seuil, 1986, p. 183.
} 
notamment par Merleau-Ponty. Or, si Castoriadis reconnaît l'existence, à la suite de Mauss, du niveau des institutions, comme l'a déjà indiqué Stéphane Vibert4, il a mis au jour un niveau plus fondamental, qui englobe les institutions particulières comme fait générique de l'institution, pensée comme auto-institution. En effet, le socialhistorique est un niveau d'être qui ne se comprend que par lui-même, sui generis, dont on ne trouve pas d'équivalent dans le monde naturel. L'institution donne lieu à ce niveau d'être inédit, prenant la forme d'un « collectif anonyme». Par cette expression, Castoriadis veut souligner qu'il ne s'agit en rien d'une subjectivité. Il suffit ici de revoir ses critiques senties de certaines notions bien connues en sociologie, qu'il s'agisse de la « conscience collectives 5 ", de l' ' $^{\text {inconscient collectif }}{ }^{6}$ » ou même des « représentations sociales 7 ». Le social-historique n'est pas plus une objectivité, au sens d'une entité distincte de l'individu, qui se tiendrait face à lui. Il s'agit d'un niveau plus fondamental, qui rend toute objectivité et toute subjectivité possibles :

[...] le social implique quelque chose qui ne peut jamais être donné comme tel. La dimension social-historique, en tant que dimension du collectif et de l'anonyme [...] est ce qui est tous et qui n'est personne [...] un non-être plus réel que tout être, ce dans quoi nous baignons de part en part, mais que nous ne pouvons jamais appréhender « en personne ${ }^{8} »$.

Cette conception du social-historique et de l'institution tend à ébranler ce que Castoriadis nomme la conception héritée de la société. Elle met entre autres à mal le réflexe de nombre de pensées sociologiques de ne s'appuyer que sur la « fonctionnalité » d'une société. Selon Castoriadis, une partie de la sociologie occulte l'effectivité de l'imaginaire en n'étudiant que ce qui relève de l'ensidique9, c'est-àdire de la dimension fonctionnelle d'une société. Or, pour Castoriadis, toute société s'auto-institue dans et par l'imaginaire : c'est là sa thèse essentielle concernant la réalité sociale. Une bonne partie de la sociologie confond ainsi, selon Castoriadis, fonctionnel et effectif, sans prendre la pleine mesure du caractère institué du fonc-

\footnotetext{
4 Stéphane Vibert, «La référence à la société comme “totalité”. Pour un réalisme ontologique de l'êtreen-société (sociologie dialectique et anthropologie holiste) », Société, automne 2006, n 26, « La sociologie de Michel Freitag », p. 87.

5 Cornelius Castoriadis, Les carrefours du labyrinthe, I, op. cit., p. 258.

6 Cornelius Castoriadis, L'institution imaginaire de la société, op. cit., p. 528.

7 Notons toutefois qu'Adams n'hésite pas à relier les significations imaginaires sociales aux représentations collectives durkheimiennes, en précisant que Castoriadis ne tient pas compte du passage, chez Durkheim, de la « conscience » aux « représentations » collectives, passage qui, selon elle, le rapproche de Castoriadis : cf. Suzi Adams, Castoriadis's ontology. Being and creation, New York, Fordham University Press, 2011, p 122.

8 Cornelius Castoriadis, L'institution imaginaire de la société, op. cit., p. 166-167. Nous soulignons.

9 Néologisme formé de la contraction de l'expression « ensembliste-identitaire », utilisé par Castoriadis pour désigner la partie du réel qui souscrit au rationnel, institué chaque fois social-historiquement, mais existant formellement, dans le monde humain, comme legein et teukhein, dimensions, respectivement, du dire et du faire social.
} 
tionnel, institution d'ordre imaginaire et non purement technique ou instrumental. Ce faisant, il y a occultation complète, de son point de vue, du fait que le fonctionnel « est agi » par la dimension imaginaire. La fonctionnalité d'une société, d'où sa rationalité procède, ne peut être saisie indépendamment de son imaginaire, au sens où les régularités sont auto-instituées par l'imaginaire d'une société :

Les innombrables régularités de la vie sociale - sans lesquelles, bien sûr, cette vie n'existerait pas - sont ce qu'elles sont parce que l'institution de cette société particulière a posé ce complexe particulier de règles, de lois, de significations, de valeurs, d'outils, de motivations, etc ${ }^{10}$.

Castoriadis soutient ainsi qu'il est impossible d'isoler les composantes fonctionnelles pour elles-mêmes, puisque la rationalité d'une société est instituée par le socialhistorique en tant que collectif anonyme.

Il y a donc " effectivité » du magma des significations imaginaires sociales dans la vie sociale. Notons ici que le terme d'effectif, qu'on retrouve très souvent sous la plume de Castoriadis, signifie ce qui est « réel », ce qui se constate et, de ce seul fait, présente des effets dans le réel. Il peut donc signifier en partie des éléments rationnels, mais également, en vertu de l'institution imaginaire des sociétés, des éléments purement irrationnels, et néanmoins agissants ${ }^{11}$. Ainsi, ce qui est effectif dans une société déborde largement la seule fonctionnalité, et plus encore : la fonctionnalité n'est telle, les régularités d'une société ne sont telles, que parce qu'elles procèdent d'une auto-institution de la société par elle-même :

Il est clair que la société ne peut exister sans une dimension fonctionnelle, mais il est aussi évident que toute société asservit sa fonctionnalité $[\ldots]$ à des finalités non pas « fonctionnelles » $[. .$.$] ,$ mais imaginaires et qui dépendent des significations sociales de l'ensemble considéré12.

Est-ce à dire qu'il y a antériorité, voire déterminisme, de l'imaginaire sur le fonctionnel, sorte de «marxisme inversé »? Castoriadis nie explicitement cette possi-

10 Cornelius Castoriadis, "La polis grecque et la question de la démocratie », dans Les carrefours $d u$ labyrinthe, II. Domaines de l'homme, Paris, Seuil, 1999, p. 330.

${ }^{11}$ C'est ainsi que Castoriadis décrit la signification imaginaire sociale principale des sociétés capitalistes : «Cette fantasmagorie réelle, ce constructum historique d'une pseudo-homogénéité effective des individus et des travaux, est une institution et création du capitalisme » (p. 349), en la caractérisant, plus loin dans son texte, comme "[c]ette signification imaginaire sociale, ce figment plus réel que toute "réalité", ce fictif effectif » (p. 352). Les significations imaginaires sociales d'une société sont effectives et réelles, bien qu'elles puissent être irrationnelles ou carrément fausses, comme Castoriadis le démontre ici à l'égard du capitalisme. $C f$. «Valeur, égalité, justice, politique : de Marx à Aristote et d'Aristote à nous ", dans Les carrefours du labyrinthe, I, op. cit., p. 325-413.

12 Cornelius Castoriadis, La création humaine, I. Sujet et vérité dans le monde social-historique. Séminaires 1986-1987, Paris, Seuil, 2002, p. 24-25. 
bilité, mais la question demeure ouverte pour les commentateurs. Il récuse à ce sujet la tentative des néokantiens, notamment, consistant à renverser l'infrastructure et la superstructure marxistes, faisant peser l'origine des déterminations d'une société non plus sur le fonctionnel, mais sur l'imaginaire et le symbolique :

Cette critique [de certains néo-kantiens] participe de la même ambiguïté que la vue marxiste qu'elle veut combattre [...]. Quant au rapport entre l'institution et la vie sociale qui s'y déroule, il ne peut pas être vu comme un rapport de forme à matière au sens kantien, et en tout cas pas comme impliquant une " antériorité » de l'une sur l'autre. Il s'agit de moments dans une structure - qui n'est jamais rigide, et jamais identique d'une société à l'autre. On ne peut pas dire non plus, évidemment, que le symbolisme institutionnel « détermine » le contenu de la vie sociale. Il y a ici un rapport spécifique, sui generis, que l'on méconnaît et déforme à vouloir le saisir comme pure causation ou pur enchaînement de sens, comme liberté absolue ou détermination complète, comme rationalité transparente ou séquence de faits bruts ${ }^{13}$.

Il ne s'agit donc pas de renverser le rapport marxiste infrastructure/superstructure, mais de renvoyer dos à dos les deux instances et les deux écoles plaidant pour la primauté de l'une sur l'autre. Il importe de penser pour lui-même ce rapport intrinsèque à toute vie humaine, de l'instituant et de l'institué, qui sont tous deux traversés par l'imaginaire. L'imaginaire d'une société, affirme-t-il, ne se laisse saisir que par les traces qu'il laisse dans et par la matérialité, ces « ombres [...] projetées sur l'agir social effectif des peuples ${ }^{14} \gg$. Il ne saurait pour autant se réduire au fonctionnel. Il y a plutôt un rapport intrinsèque et consubstantiel entre le fonctionnel et l'imaginaire. «En un sens, les outils et instruments d'une société sont des significations, ils sont la "matérialisation" dans la dimension identitaire et fonctionnelle des significations imaginaires de la société considérée ${ }^{15}$ ». Il s'agit donc de penser le rapport d'intrication de l'imaginaire et du fonctionnel : si Castoriadis sépare ces deux éléments pour l'analyse, ceux-ci sont en fait constamment liés dans la pratique. L'autoinstitution de la société fait être « des significations et des institutions, les deux toujours solidaires ${ }^{16} »$. C'est entre autres dans ce rapport entre l'imaginaire et le fonctionnel que la spécificité de la notion de «social-historique » se laisse voir, c'est-àdire dans cette "implication circulaire de la dimension identitaire [ou fonctionnelle] et de la dimension significative [ou imaginaire ${ }^{17}$ ] ». Il n'y a donc pas que matérialisation des significations en choses, objets, individus; il y a figuration et présentifica-

13 Cornelius Castoriadis, L'institution imaginaire de la société, op. cit., p. 187-188.

14 Ibid., p. 214.

15 Ibid., p. 522.

16 Cornelius Castoriadis, «Imagination, imaginaire, réflexion », dans Les carrefours du labyrinthe, $V$. Fait et à faire, Paris, Seuil, 2008, p. 272.

17 Cornelius Castoriadis, L'institution imaginaire de la société, op. cit., p. 522. 
tion ${ }^{18}$ réciproque des choses sociales (objets et individus) et des significations imaginaires sociales :

L'institution de la société est ce qu'elle est et telle qu'elle est en tant qu'elle «matérialise » un magma de significations imaginaires sociales [...]. Nous n'avons pas ici des significations « librement détachables » de tout support matériel, de purs pôles d'idéalité ; c'est dans et par l'être et l'être-ainsi de ce "support» que ces significations sont et sont telles qu'elles sont ${ }^{19}$.

La réduction classique de l'effectif au fonctionnel d'une société doit donc, pour Castoriadis, être abolie. En plus d'être la plupart du temps une projection sur l'ensemble de l'humanité de la conception occidentale de la Raison, projection dont le marxisme est sans contredit selon lui le premier coupable, cette conception on ne peut plus réductrice de l'effectivité renvoie l'imaginaire à une chimère, dans le meilleur des cas, ou l'écarte simplement. Si, donc, cette critique de Castoriadis vise en premier lieu le marxisme, elle n'épargne cependant ni Weber ni Durkheim. Ainsi, Weber, dans la perspective castoriadienne, jaugerait toute société à l'aune de la seule rationalité instrumentale, tout en prolongeant une philosophie égologique basée sur un « individualisme ontologique ${ }^{20}$ ». Durkheim, quant à lui, aurait aperçu la thèse de la réalité sui generis du social, mais n'en aurait pas tiré les conséquences épistémologiques, en ce qu'il aurait renvoyé l'imaginaire du côté des "représentations », individuelles et collectives, et serait ainsi resté pris dans une conception objectiviste de la réalité sociale.

En escamotant le niveau de l'institution imaginaire des sociétés, la sociologie se condamne à buter constamment sur cet objet qu'elle se refuse à voir. Ce faisant, elle apparaît nécessairement, aux yeux de Castoriadis, amputée d'une partie essentielle, diminuant d'autant la portée de ses explicitations. Puisque le fonctionnel se laisse saisir beaucoup plus facilement que l'imaginaire, les sciences sociales ont d'autant plus tendance, affirme Castoriadis, à prétendre pouvoir rendre compte des sociétés exhaustivement, par l'explication ou par la compréhension.

\section{Les modes de saisie du social-historique}

\section{Explication et causation}

Si la conception castoriadienne de l'institution a opéré un élargissement de l'effectivité vers l'imaginaire, on retrouve, cette fois au niveau épistémologique, un

18 Ibid., p. 514.

19 Ibid., p. 514-515. Nous soulignons.

${ }^{20}$ Cornelius Castoriadis, «Individu, société, rationalité, histoire », dans Les carrefours du labyrinthe, III. Le monde morcelé, Paris, Seuil, 1990, p. 55. 
élargissement symétrique des types d'analyse de la réalité sociale. À la conception héritée du social centrée sur la fonctionnalité correspond une conception épistémologique pour laquelle celui-ci peut être expliqué en ayant recours principalement, voire uniquement, à la causalité. L'une des idées centrales de Castoriadis consiste ici à dissocier effectivité et causation, en élargissant le spectre des relations effectives audelà de la causalité. Ainsi affirme-t-il que "[t]outes les relations effectives ne sont pas causales ${ }^{21} »$. Il y a bien, au sein de l'objet des sciences sociales, connexions, liens, rapports, mais de nature d'abord et avant tout significative plutôt que causale. Et cela tient à ce que le social-historique est le lieu par excellence où se déploient des significations imaginaires sociales. L'analyse du social-historique permet ainsi de mettre au jour des connexions non pas d'abord causales entre les phénomènes, mais bien de l'ordre des significations imaginaires sociales, soit un registre de signification spécifique au domaine social-historique et autre que celui résultant de la rationalité ensidique.

Le rapport de causalité vise l'établissement de lois dévoilant une régularité absolue entre deux phénomènes. Or, dans le domaine social-historique, pareil déterminisme est, on le sait depuis longtemps, inexistant. Non seulement parce que, comme l'ont déjà souligné Weber et, à sa suite, Aron et Passeron, parmi bien d'autres, le social ne permet pas la reproductibilité exacte d'une expérience, essentielle à l'établissement d'un " véritable » rapport causal ${ }^{22}$, mais plus encore, comme le démontre Castoriadis, parce que l'être même du social est, en très grande partie, imperméable à la causation. À vrai dire, précise-t-il, « je n'ai pas dans l'objet lui-même les conditions qui me permettraient d'établir un enchaînement causal23 ».

L'argument central avancé par Castoriadis est un argument non plus épistémologique, mais ontologique : le social-historique n'est pas appréhendable causalement de part en part. Il n'est pas suffisamment ensidique pour être compris exhaustivement de façon rationnelle, puisqu'il est d'abord et avant tout d'ordre imaginaire. S'il y a bien une part des significations et, plus largement, de la réalité sociale, tout comme de la psyché, qui est d'ordre ensidique, il est toutefois impossible de décomposer ou de

21 Cornelius Castoriadis, «Imagination, imaginaire, réflexion », dans Les carrefours du labyrinthe, $V$, op. cit., p. 272.

22 L'expression de « véritable rapport causal » soulève des difficultés, en ce qu'elle pose la question de la possibilité ou non d'une extension de la causalité au-delà du rapport causal "classique » qu'on retrouve dans les sciences pures, et qui, dans les sciences sociales, a fait le jeu du réductionnisme positiviste. Il serait intéressant de relever, à travers l'œuvre de Castoriadis, les différentes occurrences de la causalité, afin de mieux saisir s'il conservait l'idée d'un « véritable rapport causal » ou s'il croyait, au contraire, que le rapport causal était protéiforme, suivant le type d'objet auquel il s'attache. Castoriadis élargit-il la notion même de causalité ou, sans la récuser, n'en restreint-il pas plutôt la portée et les prétentions, en y adjoignant un éclairage compréhensif? Y aurait-il donc une forme d'oscillation chez lui à ce sujet? Nous y reviendrons au tout dernier point de ce texte.

23 Cornelius Castoriadis, La création humaine, I. Sujet et vérité dans le monde social-historique. Séminaires 1986-1987, op. cit., p. 29. 
recomposer entièrement le social de manière causale. Et, comme on le verra plus loin, la compréhension n'échappe pas à cette impossibilité. Il faut donc éviter de ramener toutes conditions existantes au sein du social-historique à des séquences causales : «L'existence de conditions dans une succession ne suffit pas pour en faire une succession "causale rationnelle24" ». Le fait qu'existent des relations causales dans la réalité sociale, ce que ne dénie aucunement Castoriadis, est, pour parler en ses termes, une condition «nécessaire» d'une prise en compte de l'histoire, mais non « suffisante » : toute élucidation du social-historique ne saurait donc en faire complètement abstraction ou s'y réduire.

Il importe plutôt de penser des rapports entre les éléments de la réalité sociale qui ne soient pas que causaux, voire, à certains niveaux, pas du tout, puisque non réductibles à la déterminité qu'exige l'ensidique et qui ne caractérise qu'à un degré extrêmement faible le social-historique. Le lien causal n'est pas le seul type de connexion ou de relation effective entre deux éléments de la réalité sociale ni, surtout, le plus apte à saisir le magma des significations imaginaires sociales instituées. Tout le défi consiste à penser l'entrelacement de l'imaginaire et de l'ensidique, leur part respective dans la constitution de la réalité, qui ne peut donc qu'apparaître plus précise par les ressources croisées des deux méthodes, compréhensive et explicative, tout comme de parvenir à séparer les deux dimensions, du moins, analytiquement. Castoriadis affirme que dans la pratique de la recherche, il y a nécessairement un va-et-vient entre ces deux moments, soit la recherche d'une explication/compréhension et la saisie de la spécificité de la forme créée. Il signale même "l'erreur » qu'il y a à séparer complètement ces deux "moments » de l'analyse25. La nature même de la réalité l'incline à privilégier une pluralité de méthodes. $\mathrm{Si}$ « nous ne pouvons pas renoncer à la tentative d'établir, autant que possible, les connexions et régularités "causales" ou "quasi causales" qui apparaissent dans le domaine social-historique, portées par sa dimension ensembliste-identitaire ${ }^{26} »$, il importe néanmoins d'insister sur « les limites très étroites de ce type d'approche [...] et la nécessité de tenir solidement compte, si on veut y comprendre quoi que ce soit, de tout le magma de la réalité social-historique 27 ». En définitive, on ne saurait produire une «explication» du domaine social-historique qui soit exhaustive ni proposer une analyse causale qui soit à même de fournir un portrait approfondi de la société considérée. "Toute "explication” [y] est soit triviale, soit fragmentaire et conditionnelle ${ }^{28}$. »

24 Cornelius Castoriadis, "Individu, société, rationalité, histoire », dans Les carrefours du labyrinthe, III, op. cit., p. 68.

25 Ibid., p. 54.

26 Cornelius Castoriadis, "L'imaginaire : la création dans le domaine social-historique », dans Les carrefours du labyrinthe, II, op. cit., p. 289.

27 Idem. Nous soulignons.

28 Cornelius Castoriadis, «La polis grecque et la question de la démocratie », dans Les carrefours $d u$ labyrinthe, II, op. cit., p. 330. 
Castoriadis ne rejette pourtant pas la causalité. Il soutient même, dans L'institution imaginaire de la société, que «l'histoire est par excellence le domaine où la causalité a pour nous un sens, puisqu'elle y prend au départ la forme de la motivation et que donc nous pouvons comprendre un enchaînement "causal29" ". Comment comprendre cette affirmation, et surtout le statut accordé à la causalité dans l'étude du social-historique ? Une partie du réel est d'ordre ensidique. La causalité ne saurait être complètement éliminée, afin de permettre à cette partie de la réalité d'être explicitée, mais, étant foncièrement inapte à saisir le social-historique, il y aurait donc impossibilité pour ce type d'analyse d'épuiser son objet. Il faut plutôt admettre, comme le fait Castoriadis, que «les significations bâtiss[e]nt un ordre d'enchaînements autre que, et pourtant inextricablement tissé à, celui des enchaînements de causation $3^{\circ}$ ». La position de Castoriadis est en effet claire sur ce point : il ne s'agit pas d'embrasser la compréhension contre l'explication, si nous croyons, ce faisant, parvenir à épuiser le sujet de notre étude. Il ne s'agit pas plus de rejeter la causalité, mais de rappeler son incomplétude foncière à l'égard du domaine socialhistorique, puisque celui-ci est d'abord auto-institution d'ordre non pas rationnel ou fonctionnel, mais imaginaire. La causalité n'en a pas moins une importance, en ce qu'elle permet de saisir les éléments qui relèvent de l'ensidique, contribuant ainsi à mieux comprendre les sociétés dans leur fonctionnalité. Une couche de la réalité s'y prête indéniablement, d'où le fait que la «méthode compréhensive » qu'il présente est un entrelacement de compréhension et d'explication, où celle-là prime nécessairement sur celle-ci. L'explication à elle seule ne saurait livrer une analyse fidèle de la réalité. En effet, Castoriadis n'a de cesse de préciser que la création radicale à l'origine du social-historique, si elle est immotivée, n'est pas pour autant inconditionnée. Il revient à plusieurs reprises sur cette distinction capitale entre conditionnement, immotivation et détermination. Prendre acte du caractère créateur du socialhistorique ne dispense guère de la nécessité d'expliquer ou de comprendre la nature de ces conditionnements.

Il convient d'apporter deux précisions à propos du déterminisme. Premièrement : pour Castoriadis, la création ne récuse aucunement l'existence de "déterminismes locaux, ou sectoriels ${ }^{31}$ », mais seulement la thèse d'un déterminisme "intégral et exhaustif 32 », intenable, affirme-t-il, même en physique, voire dans l'ensemble des sciences naturelles. À cet égard, bien qu'il ne soit pas ici le lieu de présenter en détail sa conception de la stratification ontologique, notons cependant que cette épistémologie plurielle trouve sa source dans une conception stratifiée, "feuilletée », de la réalité. Le pluralisme épistémologique trouve en effet chez lui sa véritable raison d'être dans la nature de la réalité, qui se compose d'une multitude de strates ou de

29 Cornelius Castoriadis, L'institution imaginaire de la société, op. cit., p. 63.

30 Ibid., p. 68.

${ }^{31}$ Cornelius Castoriadis, «Complexité, magmas, histoire. L’exemple de la ville médiévale », dans Les carrefours du labyrinthe, $V$, op. cit., p. 254.

32 Idem. 
niveaux d'être. La notion de « strate » laisse voir une certaine organisation, en même temps qu'une indéterminité essentielle, de sorte que le réel n'est ni purement inconsistant ni totalement ordonné33. Si on peut tirer du réel des ensembles délimités, d'où la possibilité d'une exploration scientifique, ou, dans le cas qui nous occupe, compréhensive et réflexive, on ne peut jamais, par la connaissance rationnelle, espérer recouvrer l'être entièrement, le "recomposer" tel qu'il se donne en lui-même, puisqu'il ne se donne qu'en partie de façon rationnelle. En somme, le réel se caractérise par " une hétérogénéité ontologique essentielle : soit, une stratification irrégulière de ce qui est; soit encore une incomplétude radicale de toute détermination entre strates de l'être/étant 34 ». S'il y a bel et bien des liens possibles et effectifs entre les différentes régions, toute visée d'unité ne saurait se faire que sur fond de différenciations régionales. Et surtout, pour notre propos, «il est clair qu'aucune théorie déterministe du social-historique ne peut prétendre à plus qu'à une validité très partielle et lourdement conditionnelle $35 »$. Ainsi, la reconnaissance de déterminations objectives à travers tout le tissu du réel, y compris à travers les fibres, indissociablement imaginaires et ensidiques, du social-historique, s'inscrit dans la conception castoriadienne de l'être non pas, a contrario de l'ensemble de la «logique-ontologie héritée », comme "déterminité », mais comme stratification ontologique essentielle et irréductible. Il y a certes une part de déterminité ensidique inhérente à l'être - à un degré autrement plus lâche quand on en vient au social-historique, en raison de son institution d'ordre imaginaire -, mais l'être en lui-même, affirme Castoriadis, ne saurait se caractériser par un déterminisme intégral, en raison de cette stratification ontologique. La pensée héritée, que réprouve Castoriadis, conçoit tout l'être et tous les étants à partir de "l'hyper-catégorie fondamentale de la déterminité 36 ", « avatar 37 » de la thèse d'un déterminisme intégral.

Deuxièmement : en réalité, loin de l'exclure, la création implique le déterminisme. Une lecture approximative et superficielle de la notion de création castoriadienne comme illusion ou simulacre pourrait laisser croire qu'elle exclut toute détermination. La création, rappelons-le, n'est pas pure indétermination, mais bien autoposition de nouvelles déterminations, comme Castoriadis le réaffirme avec force dans «Fait et à faire », en réponse à ses détracteurs : «L'idée de création est également étrangère à l'idée d'une indétermination intégrale et absolue 38 ». Si la création est position d'une forme, ce sont précisément les déterminations instituées par cette création qui

\footnotetext{
33 Nous renvoyons, dans le présent volume, au texte de Nicolas Piqué, qui explicite la notion de strate chez Castoriadis.

34 Cornelius Castoriadis, "Portée ontologique de l'histoire de la science », dans Les carrefours $d u$ labyrinthe, II, op. cit., p. 540.

35 Cornelius Castoriadis, «L'imaginaire : la création dans le domaine social-historique », dans Les carrefours du labyrinthe, II, op. cit., p. 287.

36 Ibid., p. 273.

37 Cornelius Castoriadis, «Fait et à faire », dans Les carrefours du labyrinthe, V, op. cit., p. 20.

38 Ibid., p. 21.
} 
sont le garant de l'historicité même de celle-ci, d'où l'expression de " déterminisme local ». En ce sens, « [1]e déterminisme local est impliqué par l'idée de création parce que cette création est chaque fois création d'une forme, d'un eidos déterminé39. " La création castoriadienne rompt bel et bien avec la conception de l'être comme déterminité en ce qu'elle est surgissement immotivé de déterminations. Or, ce surgissement, tout immotivé qu'il soit, ne saurait être autrement qu'en posant et instituant un niveau d'être "déterminé », mais en un sens bien précis : non pas en raison d'un causalisme intégral se rapportant uniment à l'être, mais par la signification de la forme ainsi créée, signification sui generis. En ce sens, «[1]a création implique seulement que les déterminations portant sur ce qui est ne sont jamais fermées de manière à interdire l'émergence d'autres déterminations 40 ». L'émergence, ipso facto immotivée, de déterminations, porteuse d'un nouveau rapport à l'être et d'une définition nouvelle de la temporalité, on le verra plus loin, est le fin mot de Castoriadis pour contrer la logique-ontologie héritée de la déterminité, mais non toute détermination de l'être et des étants. Ces déterminations ne sont toutefois plus génériques et universelles, vu son rejet d'un déterminisme intégral, mais toujours relatives à l'étant considéré. C'est précisément ce qu'il signifie lorsqu'il écrit: «Dans le domaine humain [...] la singularité est essentielle, c'est chaque fois une autre face de l'être de l'homme qui émerge, se crée, à travers tel individu ou telle société particuliers ${ }^{41} »$. En somme, au niveau ontologique, création et déterminations ne s'excluent pas, mais s'impliquent mutuellement, à l'instar de la compréhension et de l'explication au niveau épistémologique. Nous reviendrons sur ces aspects formels de la création historique dans le dernier point de ce texte.

Castoriadis ne délaisse donc pas la notion de causalité dans l'étude du socialhistorique. 1/ «Comprendre une société » nécessite, selon lui, comme première étape de saisie de l'ensidique, des analyses causales, notamment des recherches documentaires, une démarche qu'on pourrait qualifier, donc, d' " explicative », comprise au sens large ; 2 / Il discute également à de nombreuses reprises la notion de « causation par représentation $42 »$, notion heuristique qu'on doit à Freud, et par laquelle Castoriadis démontre que le rapport de causation s'applique aussi à la psyché, au sens où les représentations elles-mêmes, au niveau individuel, peuvent produire un effet sur le monde. Cela témoigne à la fois du fait qu'elles peuvent se coaliser dans le but d'obtenir une action, d'où l'aspect de causation par représentation psychique, mais également qu'elles ne sont pas entièrement déterminées, puisqu'elles peuvent être, dans une certaine mesure du moins, « appelées » et « choisies » par l'individu,

39 Cornelius Castoriadis, "Complexité, magmas, histoire... », dans Les carrefours du labyrinthe, V, op. cit., p. 254-255. Nous soulignons.

40 Cornelius Castoriadis, «Temps et création », dans Les carrefours du labyrinthe, III, op. cit., p. 334.

${ }^{41}$ Cornelius Castoriadis, «Anthropologie, philosophie, politique», dans Les carrefours du labyrinthe, IV. La montée de l'insignifiance, Paris, Seuil, 2007, p. 126.

42 Cornelius Castoriadis, La création humaine, I. Sujet et vérité dans le monde social-historique. Séminaires 1986-1987, op. cit., p. 151 ; p. 159, n. 1. 
qui n'est pas purement à leur merci - bien qu'il ne puisse pour autant espérer maîtriser entièrement son flux psychique. Castoriadis reconnaît donc que la causalité fait partie intégrante du monde social-historique et de la vie de la psyché, bien qu'elle n'y occupe pas la place la plus essentielle.

En somme, alors qu'on pourrait croire, a priori, que Castoriadis en appelle à une récusation de la causalité, vu l'ampleur de sa critique envers la conception déterministe de la pensée héritée, ses efforts visant la création d'une conceptualité propre à l'étude du social-historique n'excluent cependant pas la causalité - à la mesure de l'imprégnation de l'ensidique, "partout dense dans l'être», au sein du socialhistorique 43. À l'instar des dimensions ensidique et imaginaire coexistant dans la vie sociale, et selon le refus castoriadien d'une séparation entre méthode et contenu, il y a donc coordination de ce qui relève de la causalité et de ce qui peut être saisi par compréhension. Ce partage, dans le domaine social-historique, est cependant hiérarchique, puisque les connexions non causales priment, en fonction du mode d'être du social, tout autant que du but de la recherche - ainsi que nous le verrons plus loin, Castoriadis vise un "projet de compréhension totale ». La causalité demeurerait donc un outil des sciences sociales, mais périphérique - ou plutôt, une sorte de passage obligé qui livre des fruits essentiels à l'analyse, certes, mais dont on ne saurait espérer plus ou autre chose qu'une analyse des déterminismes locaux et des causations s'attachant à la partie ensidique du social-historique. Elle servirait à expliciter la part de la réalité sociale qui est effectivement ensidique : que ce soit les enchaînements d'intentions et d'actions individuelles ou encore le rapport des individus et des collectivités avec leur environnement naturel. Il n'en demeure pas moins que « le social (ou l'historique) contient le non-causal comme moment essentiel44 », et c'est pourquoi la compréhension se présente comme la part fondamentale dans l'analyse des sociétés. Ce qui relève de la dimension fonctionnelle est, précise-t-il, « subordonné à ma préoccupation centrale : restituer les significations et les institutions dans lesquelles ces significations s'incarnent, moyennant lesquelles chaque société se constitue comme société et constitue son monde propre45 ». Néanmoins, Castoriadis ne laisse jamais entendre que la compréhension ou la causalité sont souveraines et se suffisent à elles-mêmes. La compréhension ne peut en effet prétendre épuiser l'objet qu'est le social-historique, comme nous allons maintenant l'examiner.

\footnotetext{
43 Aussi affirme-t-il que « la relation déterminée, forme éminente de l'ensidique, est, comme l'ensidique lui-même, partout dense dans l'être ». Cornelius Castoriadis, "Complexité, magmas, histoire... ", dans Les carrefours du labyrinthe, $V$, op. cit., p. 255.

44 Cornelius Castoriadis, L'institution imaginaire de la société, op. cit., p. 65.

45 Cornelius Castoriadis, La création humaine, II. Ce qui fait la Grèce, 1 : D'Homère à Héraclite. Séminaires 1982-1983, Paris, Seuil, 2004, p. 49.
} 
La saisie du social-historique : création et méthode chez Castoriadis | Geneviève GENDREAU

\section{Compréhension du noyau central des significations imaginaires sociales}

La compréhension telle que la conçoit Castoriadis ne saurait, pas plus que l'explication, aboutir à une saisie complète du social-historique. Qu'est-ce donc que comprendre une société, selon Castoriadis ? Cela « signifie, d'abord et surtout, pénétrer (ou se réapproprier) les significations imaginaires sociales qui tiennent cette société ensemble $46 »$. Il faut retourner à la conception castoriadienne des significations imaginaires sociales et de la forme sous laquelle elles existent, le magma (qui, luimême, existe sous la forme d'un " magma de magmas47 »), pour y trouver la raison de l'incomplétude foncière de toute compréhension et, plus largement, de toute saisie $\mathrm{du}$ social-historique. Celle-ci provient du fait que les significations imaginaires sociales ainsi que la totalité magmatique dont elles font partie échappent, en bonne partie, à une prise d'ordre ensidique.

Le magma des significations imaginaires d'une société se compose de trois éléments. Ces « trois “vecteurs 48 " » caractérisant la singularité d'une société en sont les intentions nucléaires, les représentations (qui correspondent aux «significations » au sens strict) et les affects. Si les intentions peuvent être lues immédiatement dans les actions effectives qui se déroulent et se sont déroulées au sein de la société considérée, en rendant la restitution somme toute accessible, cela est beaucoup plus difficile en ce qui concerne les représentations, et pratiquement impossible dans le cas des affects. Et, bien entendu, ces trois vecteurs, s'il est possible de les séparer analytiquement, n'existent pas indépendamment les uns des autres dans la réalité sociale49. $\mathrm{Ni}$, d'ailleurs, au sein de la psyché elle-même, dans la mesure où le flux psychique se compose également de cette triade intentionnelle/représentationnelle/affective. Le magma des significations imaginaires sociales ne peut donc être donné à qui que ce soit en personne, qu'il s'agisse de l'individu au sein du monde vécu ou du chercheur qui tente de ressaisir l'être de la société en question - la sienne propre ou une autre société, contemporaine ou non.

Ainsi, le social-historique, en tant que collectif anonyme, transcende chaque individu. Si l'individu, affirme Castoriadis, est un « fragment ambulant de sa société50 »,

46 Cornelius Castoriadis, «La polis grecque et la question de la démocratie », dans Les carrefours $d u$ labyrinthe, II, op. cit., p. 330.

47 Cornelius Castoriadis, L'institution imaginaire de la société, op. cit., p. 336.

48 Cornelius Castoriadis, "Mode d'être et problèmes de connaissance du social-historique », dans Les carrefours du labyrinthe, VI. Figures du pensable, Paris, Seuil, 1999, p. 329.

49 Cf. Cornelius Castoriadis, «L'imaginaire : la création dans le domaine social-historique », dans Les carrefours du labyrinthe, II, op. cit, p. 329.

50 Cornelius Castoriadis, «Imaginaire et imagination au carrefour », dans Les carrefours du labyrinthe, VI, op. cit., p. 116. Ce faisant, Castoriadis déplace l'opposition classique individu/société, souvent sclérosante en sciences sociales, vers une opposition psyché/société, où l'individu (socialisé) et la société (instituante et instituée) relèvent du même niveau ontologique, soit celui de la socialité et d'un socialhistorique toujours particulier, alors que la psyché porte pour toujours la trace de sa déliaison primaire, de son asocialité foncière. 
il n'est pas pour autant en mesure de saisir le magma des significations imaginaires sociales de sa propre société :

un Athénien du $\mathrm{V}^{\mathrm{e}}$ siècle [...] ne pourrait jamais nous dire ce que c'était qu'Athènes. Il ne serait jamais qu'un individu : ce qui se réalisait dans la cité, et dans le siècle [...] tout cela transcende tout individu donné. Jamais ce n'est donné dans l'esprit de quelqu'un [...]. C'est encore d'un autre ordre lorsqu'il s'agit des significations imaginaires sociales d'une société51.

Ce niveau anonyme se donne ici à voir, du point de vue de l'individu, précisément en ce qu'il échappe à sa conscience, tout en le traversant de part en part : son aspect inconscient témoigne de sa prégnance. Cette totalité indéterminable, sans pour autant être indéterminée ${ }^{2}$, et difficilement décomposable, demeure en elle-même inaccessible au chercheur, à moins de postuler, à la manière du rationalisme et du marxisme, un point de vue absolu sur l'histoire, ce contre quoi Castoriadis n'a cessé de se battre. Il a en effet toujours récusé ce point de vue absolu, tout en maintenant par ailleurs que, dans le réel, il existe bel et bien un magma de significations imaginaires sociales qui « tient» la société ensemble.

C'est en ce sens qu'on peut mieux saisir ses quelques affirmations, qui peuvent $a$ priori sembler contradictoires avec ce qu'il avance par ailleurs au sujet de la compréhension, selon lesquelles aucune méthode n'est possible pour rendre compte du magma des significations imaginaires sociales :

il n'y a aucun moyen, aucune méthode pour trouver ce qui est le noyau vrai (et même une fois trouvé, on ne pourrait jamais démontrer que c'est cela). Car ce noyau vrai n'est jamais donné à qui que ce soit en personne. [...] Il n'y a donc ni méthodologie au sens strict, ni possibilité de "démontrer " rigoureusement quoi que ce soit; nous pouvons seulement montrer [...] que telle ou telle assertion sur telle société est une ânerie, ou très superficielle, ou laisse de côté des éléments beaucoup plus importants, etc. Dans cette recréation d'une société étrangère, le rôle essentiel appartient à l'imagination 53 .

Cette affirmation selon laquelle il ne saurait y avoir de méthode à proprement parler pour saisir les significations imaginaires sociales et, plus encore, leur « noyau

$5^{1}$ Cornelius Castoriadis, La création humaine, I. Sujet et vérité dans le monde social-historique. Séminaires 1986-1987, op. cit., p. 35.

$5^{2} \mathrm{Ou}$ encore, dans les termes de Poirier, la totalité magmatique des significations d'une société est déterminante : "il faudrait selon Castoriadis penser l'être comme autocréation, c'est-à-dire comme puissance d'auto-altération indéterminée en même temps que déterminante ». $C f$. Nicolas Poirier, « Cornelius Castoriadis. L'imaginaire radical », Revue du MAUSS, vol. 1, n 21, 2003, p. 394.

53 Cornelius Castoriadis, La création humaine, I. Sujet et vérité dans le monde social-historique. Séminaires 1986-1987, op. cit., p. 35. Nous soulignons. 
vrai », souligne non pas l'inanité des quelques indications qu'il donne quant à une démarche compréhensive, mais bien la vanité de prétendre à une saisie rationnelle exhaustive du magma des significations imaginaires sociales. L'impossibilité d'atteindre un dévoilement et une compréhension exhaustifs du magma des significations imaginaires sociales s'entend également au sens où une "vérité » indiscutable est inaccessible : les résultats de la compréhension sont toujours sujets à discussion. Dans tout processus de compréhension d'un social-historique, énonce Castoriadis :

Le « vrai » ne saurait être soumis $[\ldots]$ aux procédures ordinaires de «vérification» ou de «réfutation» qui, pense-t-on aujourd'hui $[\ldots]$, permettent de tracer une ligne de démarcation entre «science» et «non-science» $[. .$.$] . Que veut dire vrai en$ l'occurrence? Que cette idée rassemble une classe indéfinie de phénomènes historiques et sociaux [...] qui demeureraient autrement sans connexion - pas nécessairement dans leur rapport « causal» ou « structurel », mais dans leur signification; et que sa prétention de posséder un référent « réel » ou « effectif » $[. .$. peut faire l'objet d'une discussion féconde, bien [qu']interminable. En bref, elle élucide, et initie un processus d'élucidation 54 .

En somme, "l'imaginaire n'est pas un objet de savoir, il ne peut être posé comme un principe ou une cause rationnelle à partir de laquelle je pourrais déduire la réalité social-historique ; celle-ci est bien plutôt surgissement immotivé, et en tant que telle elle relève de l'imaginaire 55 ». La saisie du social-historique exige une «conversion du regard » qui rend possible une certaine monstration, mais en aucun cas, une « démonstration ».

Ainsi, l'opération compréhensive ne s'avère pas seulement d'une complexité «numérique », cherchant à restituer une infinité d'éléments et de significations, mais bien d'une complexité ontologique, puisque cette saisie intuitive du chercheur a affaire à des contenus d'ordre essentiellement imaginaire, qui dessinent des connexions au niveau de la signification, comme Castoriadis le rappelle ici. Castoriadis est donc ici conséquent, en ce qu'il affirme l'impossibilité de restituer l'entièreté du magma des significations imaginaires sociales ${ }^{56}$. Étant d'ordre imaginaire, mais, dans la mê-

54 Cornelius Castoriadis, «La polis grecque et la question de la démocratie », dans Les carrefours $d u$ labyrinthe, II, op. cit., p. 331-332.

55 Arnaud Tomès, dans Cornelius Castoriadis et Arnaud Tomès, L'imaginaire comme tel, Paris, Hermann, p. 104.

56 « [I]1 est en principe impossible de restituer, après qu'elle a été détruite, l'eidos qu'elle incarnait et réalisait [...]. C'est tout le monde des significations, des affects et des intentions - des significations imaginaires sociales - créé par ces sociétés et qui les faisaient [sic] tenir ensemble qui ne peut pas être retrouvé, mais seulement approché avec les plus grandes difficultés [...]. Ainsi chaque forme socialhistorique est-elle vraiment et authentiquement singulière; elle possède une singularité essentielle, non 
me mesure, tissé à l'ensidique d'une société, nul ne peut espérer le recouvrer entièrement, même rétrospectivement. Se fait jour ici dans toute son originalité « la tentative de Castoriadis pour penser conjointement la création et la détermination 57 ». On se trouve devant l'impossibilité logique et ontologique d'en fournir une explication, et même une compréhension exhaustive, en vertu du fait que le social-historique est en grande partie constitué de significations imaginaires sociales, lesquelles ne peuvent être saisies par les moyens de la logique ensidique. Ce n'est donc aucunement par scepticisme qu'il fait cette déclaration incendiaire, mais, pourrait-on dire, par prudence épistémique, ou plutôt, en toute conscience de la nature magmatique du social-historique.

Or, cela n'entame en rien la nécessité de postuler le social-historique comme niveau d'être sui generis au sein même de l'analyse, et d'ainsi tendre vers sa restitution. Cette affirmation selon laquelle il n'y a pas de "méthode » à même de saisir le social-historique est à entendre comme la position du cercle de la création au niveau épistémologique. On ne peut isoler une composante de la société étudiée sans la mettre en rapport avec l'ensemble des relations qui y existent, c'est-à-dire, à terme, avec la totalité de la société, en vertu du rapport circulaire, et néanmoins hiérarchique, existant entre la "forme-totale » qu'est chaque société et les éléments de celle-ci, comme on le verra plus loin. Ce rapport de circularité exige de sortir des chemins balisés, tant en philosophie qu'en sciences sociales, d'où la difficulté épistémologique, pour les sciences sociales, de rencontrer leur objet et, conséquemment, de l'examiner le plus finement possible. L'impossibilité d'appliquer une méthode permettant la saisie du social-historique réside à la fois dans cette incapacité d'ordre ontologique de saisir le «noyau central » d'une société et dans la nécessité épistémologique de le viser. Il faut tendre à restituer l'ensemble du magma des significations imaginaires sociales, tout en, paradoxalement, ne pouvant atteindre formellement un tel résultat. Cela ne signifie pas que cette démarche soit impossible pour autant, mais elle se fera avec beaucoup de précautions. À titre d'exemple, Castoriadis revient inlassablement sur les moyens d'éviter le « socio-centrisme 58 », c'est-à-dire la projection de nos propres catégories sur les autres sociétés. Ce processus est d'une grande complexité : en effet, cette projection est en partie inévitable puisque le chercheur est toujours situé à un moment précis de l'histoire. Il importe néanmoins le plus possible d'opérer un décentrement de nos catégories, sachant qu'il ne saurait être absolu. En somme, la compréhension ne peut jamais être parfaite adéquation et s'ouvre vers une démarche plus large, l'élucidation, qui souhaite prendre la pleine mesure du mode d'être, essentiellement imaginaire, mais toujours imbriqué à de l'ensidique, du social-historique.

pas numérique ou combinatoire». Cornelius Castoriadis, « Mode d'être et problèmes de connaissance du social-historique », dans Les carrefours du labyrinthe, VI, op. cit, p. 318.

57 Nicolas Poirier, L'ontologie politique de Castoriadis : création et institution, Paris, Payot, 2011, p. 26.

58 Cornelius Castoriadis, L'institution imaginaire de la société, op. cit., p. 50. 
La saisie du social-historique : création et méthode chez Castoriadis | Geneviève GENDREAU

\section{Élucidation et projet de compréhension totale}

C'est ainsi dans le cadre d'un " projet de compréhension totale59», au sein duquel les moments explicatif et compréhensif (entendu au sens strict) trouvent leur véritable raison d'être, que réside l'originalité de l'épistémologie castoriadienne des sciences sociales et historiques. Qui plus est, ce pluralisme épistémologique trouve sa source dans une conception stratifiée de la réalité, témoignant par là de sa création ontologique continue. Les deux moments que sont l'explication et la compréhension convergent vers une "élucidation » du sens même des significations, c'est-à-dire non seulement la restitution de leur contenu, mais la mise au jour de la forme même que représente chaque société.

Dans la «Préface » de L'institution imaginaire de la société, Castoriadis définit la démarche d'élucidation en l'opposant à une visée purement théorique, qui se conçoit en position d'extériorité par rapport à son objet. «Ce que j'appelle élucidation est le travail par lequel les hommes essaient de penser ce qu'ils font et de savoir ce qu'ils pensent. Cela aussi est une création social-historique ${ }^{60}$. " Bien qu'elle s'oppose à $l^{\prime}$ « illusion de la theôria ${ }^{61} »$ comme prétention explicative surplombante, l'élucidation n'en est pas moins "visée de vérité ». Elle ne prétend pas s'extraire complètement de la logique ensidique, puisque c'est encore et toujours dans ces termes, c'est-à-dire par le langage, qui est à la fois code et langue parlée, toujours particulière, qu'elle s'exprime. Mais cette visée de vérité a comme condition même le fait d'appartenir à une société. "Je peux élucider mon rapport à la langue, énonce-t-il, je ne peux pas m'en abstraire et le "regarder" - ou le "construire" de l'extérieur62. » Cette affirmation vaut, évidemment, pour toute élucidation. On retrouve ainsi, dans la préface même de l'ouvrage par lequel Castoriadis, avec toute sa verve caractéristique, rompt de manière explicite avec le marxisme, la conception selon laquelle toute pensée est un « faire social », position qu'on peut à bon droit concevoir comme reprise et réélaboration de la philosophie marxienne de la praxis. Retraçant les usages du terme dans l'histoire de la philosophie, somme toute « rares », précise-t-elle, Adams conçoit la visée d'élucidation castoriadienne comme radicalisant les courants existentialistes et prolongeant l'héritage de la phénoménologie, notamment marxiste63.

Cependant, l'élucidation au sens castoriadien est bien un projet, un travail incessant, une visée de vérité. Dans le même temps, par le fait que cette visée est une création historique, elle est un travail non seulement pratique, mais « indissociable

\footnotetext{
59 Cornelius Castoriadis, La création humaine, I. Sujet et vérité dans le monde social-historique. Séminaires 1986-1987, op. cit., p. 52.

60 Cornelius Castoriadis, L'institution imaginaire de la société, op. cit., p. 8.

61 Ibid., p. 9.

62 Cornelius Castoriadis, «Institution première de la société et institutions secondes », dans Les carrefours du labyrinthe, VI, op. cit., p. 140.

63 Cf. Suzi Adams, Castoriadis's ontology. Being and creation, op. cit., p. 26.
} 
d'une visée et d'un projet politiques 64 ». Il s'agit bel et bien d'une « autoposition », celle d'un projet politique qui est consubstantiellement projet de vérité, projet philosophique, par lequel on ne peut espérer « expliquer » le réel, la société dans le temps et dans l'espace, mais le comprendre dans une certaine mesure et, toujours, l'expliciter, l'élucider, par les moyens d'un « rendre compte et raison » radicalisé, c'est-à-dire remis en contact avec ses racines, imaginaires, comme l'une des formes qu'a prise l'exigence de signification.

Si l'élucidation est démarche " totale » puisqu'elle se sait prolongement du projet d'autonomie, elle l'est également, au point de vue épistémologique, puisqu'elle se sait reprise d'une totalité, celle du social-historique à l'étude. Castoriadis vise "l'élucidation de l'expérience social-historique totale 65 », c'est-à-dire, on l'a vu, du noyau central des significations imaginaires sociales d'une société. La notion d'élucidation rejoint ici la distinction faite plus haut entre monstration et démonstration. En effet, comme le précise Tomès, « si nous ne pouvons pas démontrer la nécessité de l'imaginaire, nous pouvons au moins l'élucider. [...] Élucider, c'est [...] éclaircir un phénomène, en rendre raison [...] en montrant en quoi ce phénomène doit être postulé66». L'essentiel de l'analyse du social-historique demeure cette élucidation, qui ne peut être exhaustive. Celle-ci vise à mettre au jour ce en quoi la société à l'étude constitue une création radicale, ce qui exige de replacer les différents éléments collectés par l'explication et la compréhension au sein de la «formetotale » en question, afin de repérer, autant que faire se peut, de quoi est fait le noyau des significations imaginaires sociales. On l'a dit, aucune analyse n'est en mesure de saisir entièrement le magma des significations imaginaires sociales d'une société, mais l'élucidation y tend, dans le cadre d'un projet de compréhension totale, sans pour autant être en mesure d'y mettre un terme définitif.

En somme, il y a restitution 1/ des éléments ensidiques par l'explication, 2/ du contenu des significations imaginaires sociales par la compréhension (comprise ici au sens strict), 3/ de ce que Castoriadis nomme le «noyau central» des significations imaginaires sociales d'une société, c'est-à-dire la saisie de l'ensemble de ces éléments (ensidiques et imaginaires) à l'intérieur de leur matrice de sens, dans le but de saisir le «sens du sens » d'une société, puisque ce qui fait sens ou non est lui-même institué67. Il s'agit de «comprendre [une société] comme forme pour elle-même et

\footnotetext{
64 Cornelius Castoriadis, L'institution imaginaire de la société, op. cit., p. 8.

65 Cornelius Castoriadis, La création humaine, I. Sujet et vérité dans le monde social-historique. Séminaires 1986-1987, op. cit., p. 368.

66 Arnaud Tomès, dans Cornelius Castoriadis et Arnaud Tomès, L'imaginaire comme tel, op. cit., p. 103.

67 Rappelons en effet que, pour Castoriadis, le réel lui-même est institué au niveau de l'imaginaire d'une société, de sorte que ce qui apparaît comme réel ou non, bon ou mauvais, important ou accessoire, n'est pas « naturel» ou universel, mais bel et bien institué. En un mot : les conditions mêmes du sens, c'est-àdire les «marqueurs » du sens, le sens même des significations imaginaires sociales, sont rendues effectives par l'institution imaginaire de la société.
} 
nouveau magma de significations instituées, pour "expliquer" et "comprendre" ce que les éléments qui lui préexistaient y sont devenus par le nouveau sens qu'ils y ont acquis $^{68} \gg$. Lorsque Castoriadis vise la restitution du noyau des significations imaginaires sociales, il pose la volonté résolue, qui se comprend réellement comme visée et projet de monstration plutôt que comme volonté de " démonstration », de la restitution du monde des significations imaginaires sociales.

\section{La création historique à l'aune du paradigme de la création/destruction ontologique}

Castoriadis suit la voie de la mise à nu de la pensée héritée, de manière à montrer l'ampleur de l'ontologie de la déterminité et ses conséquences sur la conception de l'histoire humaine et, bien entendu, sur l'épistémologie en général. Dans l'optique de Castoriadis, toute pensée qui reste en deçà de cette « déconstruction » de la déterminité paraît inopérante. Toute science sociale, on l'a vu, qui ne prend pas acte de l'action de l'imaginaire radical instituant comme étant à la source de l'institution sociale, manque la nature même de son objet. Comment s'opère cette mise à nu ? À partir de quels principes ontologiques - de quels « faits bruts », dirait-il ?

Cette conception de la création historique trouve son origine, chez Castoriadis, dans la conception du temps comme création. La distinction essentielle, au cœur de toute la pensée de Castoriadis, entre la " différence » et l' " altérité », entraîne celui-ci à élaborer une autre conception lui permettant de lier directement la temporalité et l'être. Ce faisant, il dissocie la temporalité de la spatialité, pour faire de la temporalité non pas le lieu du déploiement de l'être, mais ce déploiement de l'être lui-même comme «à-être». La temporalité n'est donc plus simple répétition ou simple « trajectoire » d'un point à un autre de l'espace, «quatrième dimension » qui ne trouve, dans cette conception héritée de la temporalité consubstantielle à la conception de l'être comme déterminité, sa singularité que "par défaut», en référence à la spatialité et avec déférence envers elle. Pour lui, « l'Être n'est pas simplement "dans" le Temps, mais il est par le Temps (moyennant le Temps, en vertu du Temps). Essentiellement l'Être est Temps [...]. Le Temps, rigoureusement parlant, est impensable sans la création; autrement, le Temps ne serait qu'une quatrième dimension spatiale surnuméraire ${ }^{69} \gg$. Cette conception de l'être comme étant fondamentalement temporalité, comprise comme à-être, attribue logiquement à toutes ses manifestations, aux étants et aux différents niveaux d'être, un même rapport de surgissement temporel. Aussi affirme-t-il que « les étants ont en eux-mêmes principe et origine de création de formes, l'être lui-même est défini par l'alloiôsis au sens fort,

68 Cornelius Castoriadis, «Individu, société, rationalité, histoire », dans Les carrefours du labyrinthe, III, op. cit., p. 68-69.

69 Cornelius Castoriadis, "L'imaginaire : la création dans le domaine social-historique », dans Les carrefours du labyrinthe, II, op. cit., p. 272. 
l'auto-altération, l'auto-création 70 ». C'est bien $d u$ fait que le temps est création que la création historique présente un double caractère, indissociable par ailleurs: immotivé et holiste. Ainsi retrouve-t-on l'idée séminale, sur laquelle il n'est pas lieu de nous pencher plus avant ici, si ce n'est pour souligner le fait que le temps et la création de l'histoire sont mus par un même principe d'immotivation : « Le temps est l'excès de l'être sur lui-même, ce par quoi l'être est toujours essentiellement àêtre $^{71} »$, dont on trouve un écho direct dans sa conception de la création historique.

Or, si son ontologie de la création apparaît clairement, le passage à l'épistémologie historique, à même d'enrichir la compréhension des modes de saisie du social-historique précédemment indiqués, demeure de prime abord énigmatique. Si, à sa défense, Castoriadis «offre un mot pour un fait 72 », force est de constater que le fait de la création, dans toute sa concrétude historique, apparaît parfois difficilement. Et ce, notons-le en passant, d'autant que se côtoient dans ses écrits, en quelque sorte à son corps défendant, vu sa critique dévastatrice de l'interprétation et de l'herméneutique ${ }^{73}$, les notions de création, certes, mais également, bien que plus rarement, ceux de « recréation », de « suite d'interprétations » et de « recréation poétique ». Faut-il pour autant en conclure, à l'instar de Rusch, qu'en dépit du fait que « [r] égulièrement reviennent des formulations plus dialectiques, qui signalent les vraies difficultés [...]. Castoriadis renonce à poser ces questions, pour privilégier une ontologie et une dramaturgie du nouveau, qui a besoin d'oppositions radicales [...] et de singularités absolues $74 »$ ? Certes, Castoriadis privilégie souvent, au moment de discuter de la création historique, des formules présentant la création comme étant " ex nihilo », mais si on s'attarde aux "formulations plus dialectiques » qu'on retrouve ici et là dans certains de ses textes, celles-ci permettent réellement de dégager, à notre sens, le rapport entre le déjà-là historique, les conditions de la création, et la création elle-même. En tous les cas, il nous semble inexact d'affirmer que Castoriadis renonce à répondre aux questions soulevées par Rusch : «Comment dès lors la création historique s'articule-t-elle à ces conditions données ? Quelle est la nature de cette relation d' "intériorité75"? " En nous basant sur certains textes de

70 Cornelius Castoriadis, «Phusis, création, autonomie », dans Les carrefours du labyrinthe, V, op. cit., p. 243.

${ }^{71}$ Cornelius Castoriadis, "Institution de la société et religion », dans Les carrefours du labyrinthe, II, op. cit., p. 470.

72 Cornelius Castoriadis, "L'imaginaire : la création dans le domaine social-historique », dans Les carrefours du labyrinthe, II, op. cit., p. 290.

73 Cf. Cornelius Castoriadis, La création humaine, II. Ce qui fait la Grèce, 1 : D'Homère à Héraclite. Séminaires 1982-1983, op. cit., p. 51-53 et La création humaine, I. Sujet et vérité dans le monde socialhistorique. Séminaires 1986-1987, op. cit., p. 48, p. 330-335, p. 359, p. 383-384, p. 391.

74 Pierre Rusch, «La vérité à l'épreuve du système. Lukàcs et le modèle esthétique », Cahiers Castoriadis, VI. Castoriadis et la question de la vérité, dir. Philippe Caumières, Sophie Klimis, Laurent Van Eynde, Bruxelles, Facultés universitaires Saint-Louis, 2006, p. 84.

75 Idem. 
Castoriadis où il s'y attelle, nous pourrons dégager plus précisément sa conception de la création historique.

\section{La création comme position de totalités}

La création est création de formes et, toujours, de « formes-totales ». Toute création est d'emblée totalisation: "la seule création qui importe est celle de formes (de lois $\left.{ }^{76}\right) »$. La position de nouvelles formes crée du nouveau, qui prend toujours la forme d'une totalité - de sens, de significations, de déterminations liées entre elles par un sens d'être inédit. Comment, plus précisément, se forme cette totalité, dans le cas d'une création historique?

Comme le souligne Bruno Bernardi, ce ne sont pas les significations imaginaires sociales qui, mises bout à bout, forment de manière purement additive une totalité. Le magma des significations imaginaires sociales d'une société est en lui-même une totalité, qui émerge d'emblée lors de sa création :

La notion à certains égards obscure de magma n'en signale pas moins un des points forts de la pensée de Castoriadis : l'insistance sur le fait que les significations imaginaires sociales d'une société ne sont pas des éléments discrets de la combinaison desquels résulterait un imaginaire social, mais inversement que celui-ci est un fait global dont ces significations (ces concepts) sont l'explicitation 77.

Bernardi met ici le doigt sur un aspect essentiel de la pensée de Castoriadis, soit le fait que la création est chaque fois création de totalités, de magmas, dont l'hétérogénéité et la stratification n'en excluent pas le caractère synthétique. Cela permet de démonter une lecture nominaliste du magma et de la forme comme purement additifs. Il n'en demeure pas moins qu'il y a, entre la forme et les significations, les « éléments » qui la composent, non pas relation d'antériorité logique ou de fondation unilatérale, mais plutôt relation indissociablement mutuelle, bien que hiérarchique, entre les significations et la nouvelle forme.

Il y a en effet relation proprement " dialectique », comme le souligne Rusch, d'interpénétration, entre la nouvelle forme et les éléments qui la composent. Donnant l'exemple de la ville médiévale, Castoriadis affirme qu'il y a "position d'une signification imaginaire sociale nouvelle - la "ville médiévale" - et d'un magma de

\footnotetext{
76 Cornelius Castoriadis, «Phusis, création, autonomie », dans Les carrefours du labyrinthe, V, op. cit., p. 241.

77 Bruno Bernardi, «En marge de Castoriadis : sur le concept d'auto-institution de la société », dans Cornelius Castoriadis et Claude Lefort : l'expérience démocratique, dir. Nicolas Poirier, Lormont, Le Bord de l'eau, 2015, p. 154. Nous soulignons.
} 
significations qui va avec celle-ci78 $»$. La position de la nouvelle forme comme "forme-totale" implique la position conjointe d'un nouveau magma de significations imaginaires sociales. Aussi affirme-t-il de la «"forme" au sens plein du terme79 » qu'elle est l'« union de l'organisation et de l'organisé8o ».

Entre la forme comme totalité et ses éléments, hétérogènes, bien que tous partie prenante du magma des significations imaginaires sociales ainsi auto-institué, il n'y a toutefois pas simple relation de "cause à effet » : il y a " excès de l'“effet" sur les "causes ${ }^{81}$ " $》$ et circularité de l'apparition de la nouvelle forme. La relation de cause à effet implique une relation où la cause mène à l'effet « sans reste », au sein de laquelle l'effet est appelé par la cause entièrement. Il s'agit plutôt, pour Castoriadis, de "mettre en lumière le caractère "intrinsèquement circulaire" de l'apparition de la nouvelle forme, et donc [...] l'impossibilité de la "produire" ou de la "déduire" d'éléments déjà donnés - car les "éléments" présupposent la forme, laquelle présuppose les "éléments"8" ».

Partant, peut-on affirmer que Castoriadis inverse la relation causale classique ? La nouvelle forme n'est pas effet, mais plutôt «cause », du moins "émergence », de nouvelles déterminations de processus non descriptibles par les niveaux d'être antérieurs. Rappelons qu'il utilise comme synonyme de l'expression « cercle de la création », qui revient à de nombreuses reprises dans ses écrits, celle de « causalité circulaire83», bien qu'il affirme sa préférence pour la première. Celle-ci laisse effectivement penser à une sorte d'inversion de la relation causale, dont on pourrait dire qu'elle devient "prospective », par opposition à rétrospective, au sens où la relation s'effectue à partir de la forme, laquelle comporte ses « raisons », son fonctionnement, ses déterminations, en elle-même. La forme n'est pas un effet ou, à tout le moins, n'est pas l'effet d'une « cause » préalable, mais plutôt l'effet de la création d'une forme sui generis. D'un point de vue ontologique, Castoriadis récuserait toutefois cette affirmation d'une simple inversion de la relation causale, puisque cette forme renouvelée de «causalité circulaire » rompt avec la double position d'identité du rapport causal «classique », qui exclut toute position immotivée d'une altérité : «identité dans la répétition des mêmes causes produisant les mêmes effets, identité

\footnotetext{
78 Cornelius Castoriadis, "Complexité, magmas, histoire...», dans Les carrefours du labyrinthe, V, op. cit., p. 267. Nous soulignons.

79 Cornelius Castoriadis, « La logique des magmas et la question de l'autonomie », dans Les carrefours du labyrinthe, II, op. cit., p. 513.

80 Idem.

81 Cornelius Castoriadis, «La source hongroise », dans Le contenu du socialisme, Paris, Union Générale d'Éditions, 1979, p. 383.

82 Cornelius Castoriadis, "Complexité, magmas, histoire... », dans Les carrefours du labyrinthe, V, op. cit., p. 254. Nous soulignons.

83 Ibid., p. 267.
} 
ultime de la cause et de l'effet ${ }^{84} \gg$. Néanmoins, il y a bel et bien une relation déterminée entre la forme et ses éléments, en vertu du fait que celle-ci

doit persister dans l'existence comme tel, ce qui requiert une relation déterminée (mais, chaque fois, à sa propre façon) entre les « états » successifs de cette forme, et aussi, puisque chaque forme est une multiplicité avec des relations déterminées (chaque fois, à leur propre façon), entre ses composantes ${ }^{85}$.

On l'a vu, chaque forme pose, à partir de ce qui est déjà-là, une singularité absolue : un nouveau sens ou niveau d'être. Si la forme devient elle-même une cause, cause d'elle-même, reprenant le déjà-là en fonction de son magma de significations imaginaires sociales, elle échappe effectivement à cette identité de la cause et de l'effet, dont on a vu qu'il est inéluctablement en excès. En somme, pour Castoriadis, l'histoire elle-même est tout sauf «circulaire» ou vaste répétition du même, puisqu'elle procède par création/destruction ontologique radicale. Toute nouvelle forme est bel et bien circulaire, du fait qu'elle est d'emblée position d'une totalité.

\section{La création comme immotivée}

Quoi qu'il en soit, cet excès de l'effet sur la cause se manifeste au sein de la formetotale en tant que telle, mais on trouve, de manière plus large, pareille relation entre la nouvelle forme et le "déjà-là » historique. Voyons en quoi se repère le caractère immotivé de la création historique chez Castoriadis.

La nouvelle forme se « découpe » sur le fond (de ce) qui était là, se détache du tissu temporel, de la surface de l'être. Bien que la métaphore spatiale soit sans doute mal avisée dans le cas de Castoriadis, vu sa critique de la pensée héritée dans laquelle la temporalité est aliénée à la spatialité, filons-la néanmoins encore un peu, quitte à ce que la fécondité s'en avère toute négative. Peut-être vaudrait-il mieux dire : un horizon, un champ, une profondeur et une durée historiques. Ou encore, pour marquer "l'action» de laquelle elle procède, une nouvelle forme émerge comme niveau d'être inédit à travers une multitude de strates, comme auto-institution. Quoi qu'il en soit, toute nouvelle forme historique se découpe, s'abstrait, s'extrait, se détache au sens où elle se pose, s'autopositionne dans l'être. Et pourrions-nous ajouter, non pour en marquer l'existence de manière négative, mais pour en démontrer la positivité : elle émerge non pas de ce qui était déjà-là, mais dans et avec. Castoriadis reconnaît ainsi la présence agissante d'une part de création et aussi de conditions, d'où l'action simultanée et non indifférenciée, au moment de l'auto-institution du socialhistorique, d'une création ex nihilo (à partir de rien), mais ni in nihilo (dans rien), ni

\footnotetext{
84 Cornelius Castoriadis, L'institution imaginaire de la société, op. cit., p. 259.

85 Cornelius Castoriadis, "Complexité, magmas, histoire... », dans Les carrefours du labyrinthe, V, op. cit., p. 255. Nous soulignons.
} 
cum nihilo (avec rien) : «le social-historique est création continuée et création dense ${ }^{86} \gg$.

La nouvelle forme historique est faite du même matériau, c'est-à-dire de significations imaginaires sociales matérialisées, comme l'est toute histoire - Castoriadis, rappelons-le, établit une équivalence entre l'histoire et le sens, l'histoire comme position de nouveau sens : «pour moi, ontologiquement, la société comme l'histoire c'est du sens ${ }^{87}$ ». Or, à partir du moment où la nouvelle forme historique se découpe, elle ne signifie plus la même chose. Elle existe en elle-même, sui generis, elle inaugure une nouvelle totalité et un niveau d'être inédit. L'aspect de totalité, une fois de plus, va de pair avec celle d'institution. L'institution est position d'une totalité.

La forme est ainsi beaucoup plus et surtout autre chose que cette abstraction : elle signifie quelque chose en elle-même. Le premier moment de ce nouveau cycle n'est jamais un effet de ce qui vient avant, mais marque plutôt l'instauration, l'institution, d'une nouvelle forme qui se détache de ce qui vient avant. On l'a dit, il y a une transformation d'ordre ontologique dans le nouveau. C'est précisément la signification de la totalité instituée qui marque l'altérité de la forme créée. Il faut donc comprendre la forme-totale comme un sens nouveau. Alors qu'il discute de l'histoire européenne, Castoriadis atteste du fait que cette création se situe au niveau des significations qu'elle institue : "...la "provenance" des composantes sociales de cette bourgeoisie, et notamment les éléments féodaux [...] a peu d'importance ; l'essentiel est que ces éléments ne se comportent plus en tant que féodaux, ni quant au pouvoir ni quant à leurs activités ${ }^{88} \gg$. La création de la nouvelle forme est autodétermination, en ce sens qu'elle vise à préserver son mode de fonctionnement, qui signe son historicité foncière.

Dans le même texte, il écrit, toujours à propos du monde occidental : « Au fur et à mesure qu'il se dégage de son héritage proprement médiéval - à la fois par ses créations propres et moyennant les "ré-interprétations" continues de son héritage qui sont fonction de celles-là -, le monde européen fait surgir deux significations imaginaires sociales et finalement s'organise sous la forme où nous le connaissons aujourd'hui 89 ». Les éléments déjà-là "sont fonction » des créations, d'où un rapport hiérarchique entre, d'une part, la nouvelle forme créée et les créations desquelles elle procède - les nouveaux "éléments" qui la composent, à savoir de nouvelles significations imaginaires sociales et, d'autre part, les « réinterprétations » de ce qui était déjà-là, qui perdurent dans la nouvelle forme. Ce rapport atteste également de

\footnotetext{
86 Cornelius Castoriadis, "Anthropologie, philosophie, politique », dans Les carrefours du labyrinthe, IV, op. cit., p. 142.

87 Cornelius Castoriadis, dans Cornelius Castoriadis et Paul Ricœur, Dialogue sur l'histoire et l'imaginaire social, Paris, Éditions de l'EHESS, 2016, p. 48-49.

88 Cornelius Castoriadis, "Complexité, magmas, histoire... », dans Les carrefours du labyrinthe, $V$, op. cit., p. 262. Nous soulignons.

89 Ibid., p. 261-262. Nous soulignons.
} 
l'hétérogénéité essentielle de toute création historique et, a fortiori, de tout étant. En effet, la création signale « une différence temporelle du surgissement, de la constitution des couches de l'objet 90 ». Cette hétérogénéité témoigne du caractère stratifié de l'être social-historique, dont on a parlé plus haut. "C'est parce qu'il y a histoire au sens fort, temporalité au sein de laquelle consécution et rupture coexistent, où il y a création au sens fort d'un nouveau qui ne "digère" pas, ni ne peut être intégralement "digéré" par, ce qui était déjà-là que l'objet effectif est magmatique91. » On touche ici à ce qui constitue un être comme être « historique ». Comment alors rendre compte et raison de ce type d'être?

On peut bien « recoller» ou « replacer» la nouvelle forme d'où on l'a extraite, dans le but d'en expliquer l'origine, en montrant que ses contours ont été abstraits de ce qu'il y avait avant, faisant la preuve qu'elle n'est ni in nihilo, ni cum nihilo. Castoriadis récuse toutefois cette façon de faire, puisque se contenter de montrer la forme découpée dans la surface déjà-là, de manière à pointer du doigt, pourrait-on dire, le «négatif» de la forme, ne permet pas de comprendre la nouvelle forme. Celle-ci, comme toute forme et toute création, est à elle-même son propre modèle, a-t-on dit : il faut donc la regarder elle-même, en tant que telle, la saisir du dedans, autant que faire se peut, ou à tout le moins à partir d'elle-même, des significations qu'elle déploie. C'est là la seule façon, en dernière analyse, d'en saisir le magma de significations imaginaires sociales, le «complexe total de ses institutions particulières ». Comme tout pour-soi, la forme historique « exige que l'on tente de [la] penser de "l'intérieur", à savoir du point de vue de son auto-constitution"2 ". Castoriadis le spécifie expressément, en référence à la ville médiévale : "la création de la ville "médiévale" [...] n'est certes pas une "nouveauté historiquement absolue" du point de vue extérieur, mais [...] à la fois par ses modes d'institution et par les significations imaginaires qu'elle porte, constitue une forme historique nouvelle93 ».

Cette rupture dans les significations instaure donc un nouveau cycle de déterminités, dont la «logique» (ou, plutôt, la cohésion et la consistance) est interne à cette nouvelle forme. La circularité même de la nouvelle forme impose son mode de saisie, endogène : on ne peut comprendre la forme qu'à partir d'elle-même. On a vu plus haut que, si le projet de compréhension totale et d'élucidation du magma des significations imaginaires sociales ne peut, en vertu de la pénétration de l'ensidique dans l'être, faire l'économie d'éléments explicatifs, on ne saurait toutefois imaginer une élucidation du social-historique par ses seuls moyens. Castoriadis renchérit :

\footnotetext{
90 Ibid., p. 258.

91 Idem. Nous soulignons.

92 Cornelius Castoriadis, «Phusis, création, autonomie », dans Les carrefours du labyrinthe, V, op. cit., p. 246.

93 Cornelius Castoriadis, "Complexité, magmas, histoire... », dans Les carrefours du labyrinthe, V, op. cit., p. 261. Nous soulignons.
} 
Pourquoi l'analyse [ensidique] échoue ? Parce que la ville médiévale est une forme social-historique que l'on peut comprendre à partir (aussi) d'elle-même - non pas « expliquer » à partir d'autre chose. Elle est création - intelligible, difficilement, en aval, non pas productible ou déductible en amont 94.

En somme, la forme est nouvelle par les significations qu'elle recèle. On ne peut espérer élucider celles-ci qu'en prenant en compte la nouvelle forme et ses modes de détermination, inédits, qui lui sont propres et véritablement en rupture avec les modes de détermination qui avaient cours jusque-là. Il y a circularité, rupture, "position de nouvelles déterminations $95 »$. Le champ social-historique, pour Castoriadis, se définit par sa créativité. La création social-historique au sens fort réside dans l'abstraction, sur le réel, d'un nouveau «cycle» de déterminations. La nouvelle forme s'abstrait du déjà-là, partiellement, pour marquer, dans le temps, ou plutôt : temporellement, son institution - au sens où elle représente une rupture temporelle au sein du social-historique, une sorte de déchirement, de déchirure de (plutôt que " dans ", comme l'être est ce temps et ses émergences, cette temporalisation même) la temporalité. Bref, toute création est non pas dans le temps, mais est Temps; le temps et l'histoire sont création tout autant que destruction.

\section{Conclusion}

Partie de la critique castoriadienne de la conception de la société, particulièrement en sciences sociales, nous avons voulu souligner le geste castoriadien d'élargissement de l'effectivité social-historique pour y inclure non seulement les éléments ensidiques et fonctionnels, mais également les éléments jugés «irrationnels » ou « irréels », découlant de l'imaginaire social. Plus encore, il s'est agi de montrer toute la radicalité de ce geste de Castoriadis, en ce qu'il retourne à la racine même du social, pour faire voir que l'institution des sociétés, entendue ici comme niveau générique et fait anthropologique, réside, à proprement parler, et dans une indéniable positivité, dans leur imaginaire. Castoriadis met ainsi de l'avant ce "fait massif» d'une création des sociétés à partir de ce qui a semblé, pour l'ensemble de la pensée héritée, au mieux, un épiphénomène, dans le pire des cas, un élément irrémédiablement marqué du sceau d'une incomplétude ontologique irréductible. Il

inverse la perspective héritée afin d'en montrer l'impensé, c'est-à-dire l'autoposition sociétale d'ordre imaginaire plutôt que simplement fonctionnelle et, plus encore, le lien indissociable, traversant toute société et toute psyché, entre l'ensidique et l'imaginaire.

94 Ibid., p. 267.

95 Cornelius Castoriadis, «Fait et à faire», dans Les carrefours du labyrinthe, V, op. cit., p. 20. 
Les rapports de la raison et de l'imaginaire sont sous-tendus par le principe selon lequel « il y a irréductibilité de la signification à la causation 96 ». On retrouve la manifestation de ce principe à tous les niveaux chez Castoriadis. D'abord, au niveau ontologique, il donne lieu à une relation de circularité entre fonctionnel et imaginaire, ainsi qu'entre le déjà-là et le créé, mettant au jour le "cercle de la création ». Ce rapport se retrouve tel quel dans l'épistémologie castoriadienne : aussi avons-nous vu que Castoriadis ne rejette nullement la causalité, ni même le déterminisme, mais qu'il les remet en quelque sorte «à leur place », évaluant la part de l'être du socialhistorique qu'elles sont en mesure d'éclairer. Ainsi, la causalité s'applique à la partie ensidique de la réalité sociale et la compréhension au sens strict pallie les manques de celle-ci, en se concentrant sur le contenu des significations imaginaires sociales. Ces aspects compréhensif et explicatif de l'analyse du social-historique convergent vers un "projet de compréhension totale», s'appuyant sur l'élucidation. Ce projet dépasse alors l'épistémologie pour se transposer sur le terrain proprement politique, celui du projet d'autonomie et de ses conditions d'avènement et de pérennité.

L'originalité de l'apport de Castoriadis pour les sciences sociales se dessine ainsi dans l'entrelacement des différents moments et dans leur hiérarchisation, au profit d'un type d'analyse qui dépasse les fonctions strictement logiques de la raison pour prendre en compte l'entièreté de la réalité social-historique, soit la triade représentationnelle, affective et intentionnelle qu'elle constitue, sous la forme d'un magma de significations imaginaires sociales. Elle se donne également à voir au plus haut degré dans sa volonté ferme de penser ensemble ce projet d'élucidation comme méthode philosophique et politique, ainsi que le paradigme de la création/destruction ontologique. Ainsi, la création historique apparaît dans toute sa complexité : ses caractères principaux et principiels révèlent ce rapport inextricable de l'ensidique et de l'imaginaire, de l'institué et de l'instituant, au sein même du social-historique. La création, d'un côté, n'abolit pas les réinterprétations continues : la création d'une nouvelle forme "coexiste», pourrait-on dire, avec la reprise d'un héritage. En d'autres termes : la création, si elle est ex nihilo, n'est ni in nihilo, ni cum nihilo, ou encore, la création implique le déterminisme. En effet, toute création est autoposition de nouvelles déterminations; mais le fait de la création témoigne de l'indétermination même de l'être, indétermination à la mesure, précisément, de l'existence de créations ontologiques et, dans le cas qui nous occupe, historiques. De l'autre côté, s'il y a bien coexistence entre le nouveau et le déjà-là, se présentant sous la forme d'un processus d'interprétations continues d'un donné historique et culturel, la neutralité du terme de « coexistence » ne doit pas masquer le rapport hiérarchique qui existe entre les deux. En effet, cesdites réinterprétations dépendent des créations. En somme, c'est en posant la création ontologique radicale, avec ses implicationsau niveau épistémologique, que Castoriadis en arrive à formuler une redéfinition de la raison.

96 Cornelius Castoriadis, L'institution imaginaire de la société, op. cit., p. 68. 